\title{
Resonant coherent Bragg rod analysis of strained epitaxial heterostructures
}

\author{
D. P. Kumah, ${ }^{1, a)}$ A. Riposan, ${ }^{1}$ C. N. Cionca, ${ }^{1}$ N. S. Husseini, ${ }^{1}$ R. Clarke,${ }^{1}$ J. Y. Lee ${ }^{2}$ \\ J. M. Millunchick, ${ }^{2}$ Y. Yacoby, ${ }^{3}$ C. M. Schlepütz, ${ }^{4}$ M. Björck, ${ }^{4}$ and P. R. Willmott ${ }^{4}$ \\ ${ }^{1}$ Applied Physics Program, Physics Department, University of Michigan, Ann Arbor, Michigan 48109, USA \\ ${ }^{2}$ Materials Science and Engineering Department, University of Michigan, Ann Arbor, Michigan \\ 48109, USA \\ ${ }^{3}$ Racah Institute of Physics, Hebrew University, Jerusalem 91904, Israel \\ ${ }^{4}$ Swiss Light Source, Paul Scherrer Institut, CH-5232 Villigen PSI, Switzerland
}

(Received 27 June 2008; accepted 4 August 2008; published online 26 August 2008)

\begin{abstract}
The resonant response of the complex x-ray scattering factor has been used in conjunction with the coherent Bragg rod analysis phase-retrieval algorithm to determine the composition and strain profiles of ultrathin layers of GaAs grown on InGaAs buffers. The buffer layers are nominally latticed matched with the InP substrate and the subsequent GaAs growth is compared at two different temperatures: 480 and $520^{\circ} \mathrm{C}$. We show that electron density maps extracted from Bragg rod scans measured close to the Ga and As $K$-edges can be used to deconvolute roughness and intermixing. It is found that indium incorporation and roughening lead to a significant reduction of the strain in this system. (C) 2008 American Institute of Physics. [DOI: 10.1063/1.2975835]
\end{abstract}

Lattice mismatch strain is ubiquitous in heteroepitaxial semiconductor systems with many benefits but also technical challenges for device applications. ${ }^{1}$ Processes such as island formation and interdiffusion leading to the relaxation of strain may result in considerable compositional and morphological modifications. A typical example is the formation of self-assembled quantum dot structures.

$\mathrm{X}$-ray probes are an excellent tool for characterizing these systems: they are nondestructive and have a high penetration power for studying buried structures and interfaces. Morever, the ability to tune $\mathrm{x}$-ray energies at synchrotron sources also enables element specificity for quantitative studies of intermixing in self-assembled coherently strained systems. $^{2-5}$

In this letter, we present results from an x-ray technique that combines the energy dependence of the atomic scattering factor and the subangstrom resolution of atomic species obtained from the recently developed model-independent coherent Bragg rod analysis (COBRA) (Ref. 6) phase-retrieval algorithm. We have used this approach to study in fine detail the vertical composition and strain profiles of ultrathin GaAs layers on an InGaAs buffer lattice matched with InP. This is a moderately strained system in which strain relaxation is accompanied by roughening of the surface. ${ }^{7-9}$ For a complete determination of the structural relaxation mechanisms a detailed study of the strain and chemical composition of these roughened films is essential.

COBRA has been applied successfully to provide subangstrom-resolution information on several epitaxial systems ${ }^{10,11}$ with in-plane periodicity and out-of-plane aperiodicity. The structure of such a system can be determined from complex scattering factors (CSFs) measured along substrate-defined Bragg rods. The measured x-ray intensity provides the amplitude of the CSF but not the phase. The COBRA method is used to retrieve the phase information. The Fourier transform of the COBRA-determined CSFs provides a three-dimensional electron density (ED) distribution

${ }^{\text {a)} E l e c t r o n i c ~ m a i l: ~ d k u m a h @ u m i c h . e d u . ~}$ function for the system with Gaussian-like peaks representing atomic sites. The structure of the system can be determined from the positions of the ED peaks with subangstrom resolution. The volume integral of these peaks is proportional to the x-ray energy-dependent effective EDs of the atomic species occupying those positions. A normalization factor is usually obtained relative to the ED peaks deep inside the substrate where there are no deviations from the nominal bulk structure to determine the chemical distribution in the system.

In the vicinity of the interfaces and close to the top surface where the composition and coverage may not be known a priori, it is a nontrivial matter to determine which elements contribute to the ED peak and what their relative fractions are. To resolve this ambiguity, we compare COBRAdetermined ED maps measured at different $\mathrm{x}$-ray energies corresponding to absorption edges of one or more of the specific atomic species comprising the ED peaks. For the case of the GaAs/InGaAs/InP system described here, we performed Bragg rod measurements close to and well above the $\mathrm{Ga} K$-edge. The effective scattering power for $\mathrm{Ga}$ varies by about $20 \%$ across the Ga $K$-edge. The difference in the energy-dependent ED maps permits an accurate determination of the relative concentrations of Ga and In as a function of the distance from the interface, independent of the effects of incomplete monolayer (ML) coverage. This makes the resonant-COBRA technique particularly suitable for alloy systems exhibiting significant compositional intermixing and interfacial/surface roughness. Here, we report on the observation from the vertical concentration profiles that, on deposition of two MLs of GaAs on the InGaAs buffer, an interplay between In alloying and surface coarsening leads to a significant reduction of strain.

The samples were grown using solid-source molecular beam epitaxy using $\mathrm{As}_{4}$. The composition, deposition rate $\left(R_{\mathrm{Ga}}\right)$ and As flux $\left(F_{\mathrm{As}}\right)$ were calibrated using reflection high energy electron diffraction oscillations, and the growth temperature was measured using an optical pyrometer. ${ }^{9}$ $\mathrm{In}_{0.53} \mathrm{Ga}_{0.47} \mathrm{As}$ buffers were grown lattice matched to $\operatorname{InP}(001)$ substrates at $480{ }^{\circ} \mathrm{C}$, followed by the growth of 


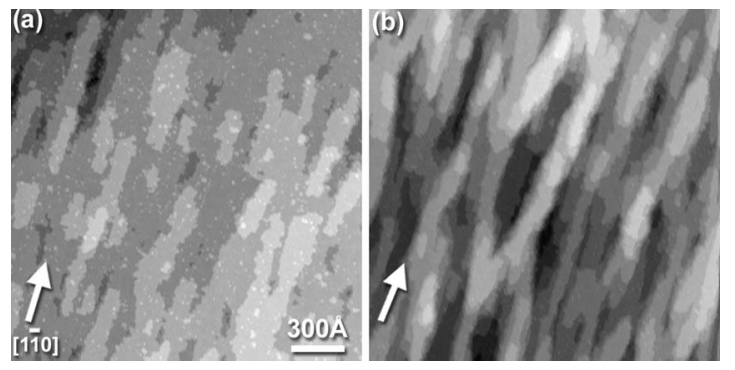

FIG. 1. STM images for 2 ML thick GaAs films on lattice-matched $\mathrm{In}_{0.53} \mathrm{Ga}_{0.47} \mathrm{As} / \mathrm{InP}$ grown at (a) 480 and (b) $520^{\circ} \mathrm{C}$ (Ref. 9).

two MLs of GaAs at either $480{ }^{\circ} \mathrm{C}\left(R_{\mathrm{Ga}}=0.05 \mathrm{ML} / \mathrm{s}, F_{\mathrm{As}}\right.$ $=1.5 \mathrm{ML} / \mathrm{s})$ or $520{ }^{\circ} \mathrm{C}\left(R_{\mathrm{Ga}}=0.20 \mathrm{ML} / \mathrm{s}, F_{\mathrm{As}}=1.5 \mathrm{ML} / \mathrm{s}\right)$. For the latter film, the buffer was heated to the desired temperature over 5 min under an $\mathrm{As}_{4}$ flux prior to the deposition of the film.

A detailed analysis of the sample growth and morphological evolution of tensile-strained GaAs films on InGaAs/InP has been recently described. ${ }^{9}$ The rms roughness of these strained films increases with increasing growth temperature from $0.17 \mathrm{~nm}$ at $480{ }^{\circ} \mathrm{C}$ to $0.35 \mathrm{~nm}$ at $520{ }^{\circ} \mathrm{C}$. At $480{ }^{\circ} \mathrm{C}$ [Fig. 1(a)] the surface exhibits two-dimensional islands in addition to a terrace structure similar to that of the buffer. At $520{ }^{\circ} \mathrm{C}$ [Fig. 1(b)], the terrace structure is obscured and the morphology consists of narrow mesas and troughs. The roughening of these films has been attributed to an increase in the group-III adatom population due to the desorption of As from the surface. ${ }^{9}$

Surface diffraction experiments were carried out at the Materials Science beamline at the Swiss Light Source, Paul Scherrer Institut, Switzerland. For each sample, 12 symmetry-inequivalent rods and four equivalent rods were obtained at 10.365 and $11.865 \mathrm{keV}$, with an energy resolution $d E / E=10^{-4}$. The energies were selected to be $2 \mathrm{eV}$ below the Ga and As absorption edges, respectively, to take advantage of the anomalous dispersion of the respective atomic structure factors with x-ray energy. Diffraction intensities were recorded using a Pilatus $100 \mathrm{~K}$ pixel detector with appropriate energy threshold settings to suppress the detection of fluorescent photons from the sample. ${ }^{2}$ Samples were placed in an evacuated chamber with a beryllium window during data collection to prevent oxidation. The angle of incidence of the incoming beam was fixed close to the InGaAs critical angle to eliminate the contribution of the InP substrate to the diffraction intensity.

For both samples measured, an ED map was obtained at each of the selected energies using COBRA. The differences in the ED maps were used to determine, quantitatively, the composition of the two systems.

Each layer in the system can be expressed as $\operatorname{In}_{x} \mathrm{Ga}_{y} \mathrm{As}_{f_{z}}$ where $x$ and $y$ are the respective fractional contributions of In and $\mathrm{Ga}$, and $f_{z}$ is the fractional occupancy of that layer. At each energy, the vertical profile of $f_{z}$ can be obtained by directly normalizing the COBRA-derived As ED peaks to the expected effective number of electrons for As at the respective energy. For each sample, we find that $f_{z}$ calculated both at the $\mathrm{Ga}$ and As edges are equivalent, indicating that there is no intermixing between the group-III and group- $\mathrm{V}$ sublattices, i.e., there is an insignificant density of antisite defects. We compare $f_{z}$ extracted from the scanning transmission microscopy (STM) images in Fig. 1 with $f_{z}$ obtained indepen-

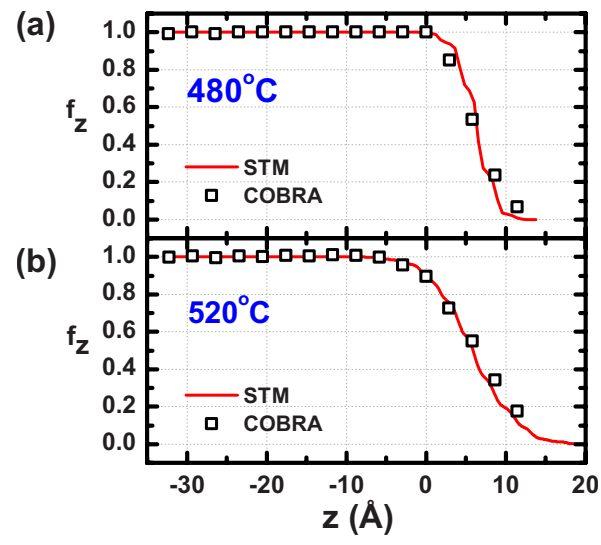

FIG. 2. (Color online) COBRA-determined vertical fractional occupancy, $f_{z}$, profiles for a $2 \mathrm{ML} \mathrm{GaAs} / \mathrm{InGaAs}$ system grown at (a) 480 and (b) $520^{\circ} \mathrm{C}$. Occupancy profiles extracted from STM images in Fig. 1 are shown for comparison. Note that $z=0$ is the nominal position of the interface.

dently by COBRA for the samples grown at $480{ }^{\circ} \mathrm{C}$ [Fig. 2(a)] and $520^{\circ} \mathrm{C}$ [Fig. 2(b)]. The regions with $f_{z}<1$ correspond to roughness at the film surface. The profiles determined by STM and COBRA are in good agreement, with any differences attributed to the different effective probe areas, and to the fact that the topmost layers with low occupancy observed by STM contribute little to the x-ray intensities.

The results obtained by COBRA are averaged layer-bylayer over the coherence length of the x-ray beam, which is on the order of $500 \mathrm{~nm}$. Thus, the analysis is not sensitive to lateral variations in composition and lattice spacing. However, we may assume in-plane uniformity in composition and lattice spacing in interpreting the observed structure of the system since excellent film/buffer registry is expected for ultrathin films.

The amount of In and Ga in each layer is determined as follows. For each group-III layer comprising both In and $\mathrm{Ga}$, let $T_{10}$ and $T_{11}$ be the total ED for that layer obtained from COBRA at 10.365 and $11.865 \mathrm{keV}$, respectively. We can now separate $x$ and $y$ at the two energies as follows:

$$
\begin{aligned}
& T_{10}=x \operatorname{In}_{10}+y \mathrm{Ga}_{10}, \\
& T_{11}=x \mathrm{In}_{11}+y \mathrm{Ga}_{11},
\end{aligned}
$$

where the subscripts 10(11) refer to the corresponding atomic scattering factors for In and $\mathrm{Ga}$ at $10.365 \mathrm{keV}$ $(11.865 \mathrm{keV})$. It is straightforward to solve algebraically for $x$ and $y$.

The film composition profiles shown in Fig. 3 for 480 and $520{ }^{\circ} \mathrm{C}$ are obtained by normalizing $x$ and $y$ to the

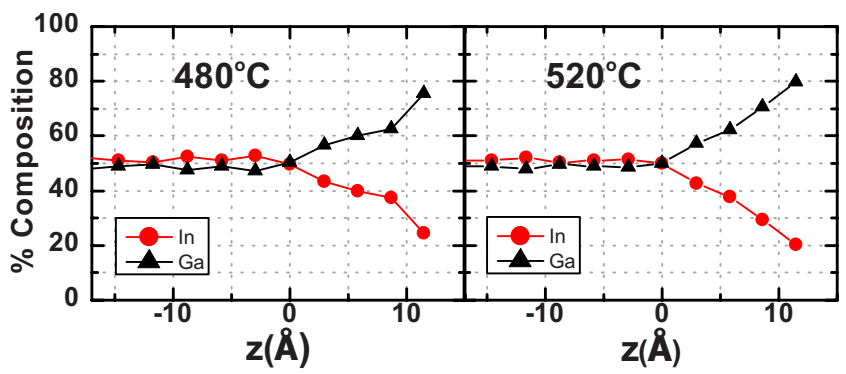

FIG. 3. (Color online) In and Ga composition profiles for the nominal $2 \mathrm{ML}$ $\mathrm{GaAs} / \mathrm{InGaAs}$ system grown at 480 and $520^{\circ} \mathrm{C}$ determined using resonant COBRA. 


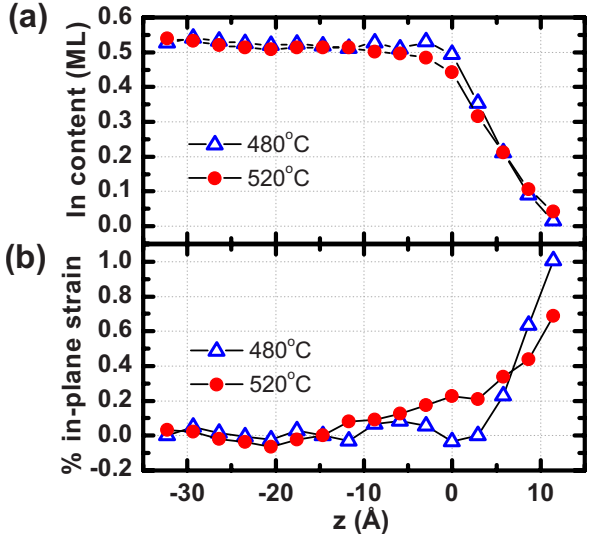

FIG. 4. (Color online) COBRA-determined (a) In content profiles and (b) in-plane strain profiles for the nominal $2 \mathrm{ML} \mathrm{GaAs/InGaAs} \mathrm{system} \mathrm{grown}$ at $480^{\circ}$ and $520^{\circ} \mathrm{C}$. In-incorporation into the nominal GaAs film effectively reduces the film/buffer mismatch strain.

COBRA-determined values for $f_{z}$ for the two growth temperatures. The composition deep in the buffer for both samples is $\operatorname{In}_{0.53} \mathrm{Ga}_{0.47} \mathrm{As}$ as expected; however, both samples show significant In-incorporation into the nominal GaAs film. The In composition of the films at both growth temperatures in Fig. 3 is about $40 \%$ near the film/buffer interface and close to $20 \%$ at the surface.

The In content, $x$, as a function of depth is compared for the two growth temperatures in Fig. 4(a). By integrating the In content for the two samples, we observe that the $520^{\circ} \mathrm{C}$ sample contains a net $0.19 \mathrm{ML}$ less In than does the $480{ }^{\circ} \mathrm{C}$ sample, indicative of enhanced In desorption at $520^{\circ} \mathrm{C}$. Using the previously reported ${ }^{13}$ Arrhenius-type dependence of the desorption rate, we can estimate the amount of In desorbed at 480 and $520{ }^{\circ} \mathrm{C}$. For a prefactor $\nu=2.6 \times 10^{13} \mathrm{~s}^{-1}$ and desorption activation energy $E_{D}=2.57 \mathrm{eV},{ }^{13}$ the calculated amount of In desorbed at $480{ }^{\circ} \mathrm{C}$ is $0.03 \mathrm{ML}$, and 0.2 $\mathrm{ML}$ at $520^{\circ} \mathrm{C}$, consistent with our experimental values.

Accounting for the Poisson ratio of InGaAs, we can estimate the strain in each ML, based on the measured composition and out-of-plane lattice spacing, assuming heteroepitaxial growth. Figure 4(b) shows profiles of the in-plane strain at the two growth temperatures. The strain is zero deep in the buffer, as expected. Both films are in tension with the strain gradient being more gradual for the $520{ }^{\circ} \mathrm{C}$ sample with a maximum strain of $0.68 \%$ compared with $1.01 \%$ for the $480{ }^{\circ} \mathrm{C}$ sample. Note that, in the absence of Inincorporation, a much larger strain $(3.7 \%)$ is expected based on the nominal lattice mismatch between the buffer and GaAs.

An In-rich surface has been shown previously to arise during the growth of InGaAs due to In surface segregation. ${ }^{14}$ During the subsequent growth of the GaAs film, the results in Fig. 4 show that In-incorporation from the InGaAs surface into the film drastically reduces the mismatch strain from $3.7 \%$ to less than $1.0 \%$ by expanding the unit cell size. Inincorporation into the film is highest closest to the interface, leading to the depletion of the buffer surface. This causes the observed strain in the buffer and a greater relaxation of misfit strain at the interface than at the film surface. Further relaxation occurs at the surface for higher film growth temperatures due to roughening.

The technique presented here provides valuable information about the chemical composition and distribution of strain in ultrathin films of tensile-strained GaAs/ $\mathrm{In}_{0.53} \mathrm{Ga}_{0.47} \mathrm{As}$ for two growth temperatures. In-incorporation into the film serves as an effective mechanism for strain relaxation in this system.

This work was supported in part by the US National Science Foundation under Grant No. DMR-0606048.

${ }^{1}$ C. Teichert, Phys. Rep. 365, 335 (2002).

${ }^{2}$ M. Hanke, D. Grigoriev, M. Schmidbauer, P. Schäfer, R. Köhler, R. L. Sellin, U. W. Pohl, and D. Bimberg, Appl. Phys. Lett. 85, 3062 (2004).

${ }^{3}$ L. N. Coelho, R. Magalhës-Paniago, A. Malachias, J. G. Zelcovit, and M. A. Cotta, Appl. Phys. Lett. 92, 021903 (2008).

${ }^{4}$ A. Malachias, S. Kycia, G. Medeiros-Ribeiro, R. Magalhäes-Paniago, T. I. Kamins, and R. S. Williams, Phys. Rev. Lett. 91, 176101 (2003).

${ }^{5}$ I. Kegel, T. H. Metzger, A. Lorke, J. Peisl, J. Stangl, G. Bauer, J. M. Garcia, and P. M. Petroff, Phys. Rev. Lett. 85, 1694 (2000).

${ }^{6}$ Y. Yacoby, M. Sowwan, E. Stern, J. O. Cross, D. Brewe, R. Pindak, J. Pitney, E. M. Dufresne, and R. Clarke, Nat. Mater. 1, 99 (2002).

${ }^{7}$ P. Krapf, Y. Robach, M. Gendry, and L. Porte, J. Cryst. Growth 181, 337 (1997).

${ }^{8}$ Gendry, G. Grenet, Y. Robach, P. Krapf, L. Porte, and G. Hollinger, Phys. Rev. B 56, 9271 (1997).

${ }^{9}$ J. Lee, C. Pearson, and J. Millunchick, J. Appl. Phys. 103, 104309 (2008).

${ }^{10}$ C. N. Cionca, A. Riposan, D. P. Kumah, N. S. Husseini, D. A. Walko, Y Yacoby, J. M. Millunchick, and R. Clarke, Appl. Phys. Lett. 92, 151914 (2008).

${ }^{11}$ P. R. Willmott, S. Pauli, R. Herger, C. M. Schlepütz, D. Martoccia, B. D. Patterson, B. Delley, R. Clarke, D. Kumah, C. Cionca, and Y. Yacoby, Phys. Rev. Lett. 99, 155502 (2007).

${ }^{12}$ C. M. Schlepütz, R. Herger, P. R. Willmott, B. D. Patterson, O. Bunk, C. Brönnimann, B. Henrich, G. Hülsen, and E. F. Eikenberry, Acta Crystallogr., Sect. A: Found. Crystallogr. A61, 418 (2005).

${ }^{13}$ Ch. Heyn, A. Bolz, T. Maltezopoulos, R. L. Johnson, and W. Hansen, J. Cryst. Growth 278, 46 (2005).

${ }^{14}$ A. Riposan, J. M. Millunchick, and C. Pearson, J. Vac. Sci. Technol. A 24, 2041 (2006). 